\title{
Temsirolimus in the treatment of relapsed or refractory mantle cell lymphoma
}

This article was published in the following Dove Press journal:

OncoTargets and Therapy

4 September 2010

Number of times this article has been viewed

\author{
Nousheen Samad' \\ Anas Younes ${ }^{2}$ \\ 'Division of Pharmacy, Pharmacy \\ Clinical Programs, ${ }^{2}$ Department \\ of Lymphoma and Myeloma, The \\ University of Texas MD Anderson \\ Cancer Center, Houston, TX, USA
}

Correspondence: Anas Younes Department of Lymphoma and Myeloma, The University of T MD Anderson Cancer Center, 1515 Holcombe Blvd, Houston, TX 77030, USA

$\mathrm{Tel}+\mathrm{I} 7137454256$

Fax +I 7I3 7945656

Email ayounes@mdanderson.org
Abstract: Mantle cell lymphoma (MCL) is a rare and aggressive subtype of lymphoma associated with a poor prognosis. Chemotherapy is the mainstay of frontline treatment for patients with this disease. Despite high response rates to combination chemotherapy regimens, the majority of patients relapse within a few years of treatment. Therefore, finding efficacious treatments for relapsed or refractory disease has become a growing area of clinical research. The mammalian target of rapamycin (mTOR) is responsible for integrating cell signals from growth factors, hormones, and nutrients and communicating energy status. Scientific research on aberrant molecular pathways in cancer has revealed that several proteins along the mTOR pathway may be upregulated in this and other types of lymphoma. Temsirolimus is the first mTOR inhibitor that has shown clinical efficacy in treating MCL that has relapsed after frontline treatments.

Keywords: rapamycin, CCI-779, MCL, mTOR, PI3K, Akt

\section{Current practices in managing mantle cell lymphoma Introduction}

Mantle cell lymphoma (MCL) is an uncommon subtype of non-Hodgkin lymphoma (NHL). Despite having been identified as a clinically and morphologically unique type of lymphoma 20 years ago, MCL lacks a standard-of-care regimen in current treatment approaches. Although efficacious frontline treatments with combination chemotherapy regimens have been identified, the rate of relapse remains unacceptably high. A growing body of research suggests targeted therapies hold promise for patients with relapsed or refractory disease. Among these new targeted agents is a class of drugs called mammalian target of rapamycin (mTOR) inhibitors. Temsirolimus, the first drug in this class marketed as an anticancer agent, has shown in vitro and in vivo activity against MCL. This review of the literature will discuss the application and use of temsirolimus in relapsed or refractory MCL.

NHL is the most commonly seen hematologic malignancy in the United States. According to the American Cancer Society, it is the fourth leading cause of cancer in women and the fifth leading cause of cancer in men in the United States; an estimated 65,980 people were diagnosed with the disease in 2009. NHL encompasses a large and heterogeneous group of lymphomas, each with unique pathologic and clinical characteristics. Among the more than 30 subtypes of NHL, MCL accounts for $6 \%$ of cases and carries the worst prognosis. ${ }^{1}$ 
MCL is an aggressive lymphoma with a short overall survival (OS) duration when left untreated; the median OS duration is $3-5$ years. ${ }^{2-6}$ The majority of patients have progressed to advanced stages (III or IV) of the disease by the time of diagnosis and often have disease outside of the lymph nodes. ${ }^{3}$ The most frequent sites of extranodal involvement are the bone marrow, peripheral blood, and gastrointestinal tract. ${ }^{4}$ The disease has a slight male predominance, and patients are typically in their seventh decade of life at the time of diagnosis. ${ }^{5}$

Specific clinical features have been identified as prognostic factors and are used to calculate a prognostic score called the Mantle cell International Prognostic Index (MIPI). ${ }^{7}$ Indicators of poor prognosis (low performance status, advanced age, high lactate dehydrogenase levels, and leukocytosis) are assigned values and added together. The corresponding score may be useful for predicting the clinical course of an individual's disease and has the potential to help guide treatment.

\section{Newly diagnosed MCL}

In a very small subset of patients, MCL has an indolent course. A watch-and-wait method has been used in these patients and may be reasonable as long as their disease progression remains slow. ${ }^{8}$ For the majority of patients with MCL, however, the prompt initiation of cytotoxic therapy is warranted. Chemotherapy is the mainstay of frontline treatment, and the addition of biologic therapy (eg, rituximab) has become a widely accepted standard. Consolidation treatment using high-dose chemotherapy followed by autologous hematopoietic stem cell transplantation is now a growing area of clinical research in this setting.

Current options for frontline therapy include combination regimens, usually involving multiple conventional chemotherapy agents, rituximab, and corticosteroids. Early data with rituximab, cyclophosphamide, doxorubicin, vincristine, and prednisone (R-CHOP) therapy demonstrated favorable initial responses in patients with MCL, but not the increase in survival that was demonstrated in other subtypes of NHL using the same regimen. ${ }^{9}$ A prospective single-center study assessed the efficacy of a more dose-intense regimen involving rituximab, cyclophosphamide, vincristine, doxorubicin, and dexamethasone alternating with methotrexate and cytarabine (R-hyperCVAD/MA).$^{10}$ The authors of the latter trial concluded that dose intensification does increase response rates and has the potential to increase OS. ${ }^{11}$ More recently, a prospective multicenter study by the Southwest Oncology Group attempted to replicate these results and confirmed the high response rates seen in the single-center study. ${ }^{12} \mathrm{~A}$ newer approach in the frontline setting involves studying the combination of R-hyperCVAD/MA with targeted therapies like bortezomib, a proteosome inhibitor approved for relapsed MCL, with the hopes of increasing first-line efficacy. Studies of this combination are currently underway.

One limitation of the R-hyperCVAD/MA regimen is the high rate of adverse events associated with the MA cycles, especially in patients older than 60 years. ${ }^{10}$ In an attempt to decrease toxicity while maintaining efficacy with this regimen, the Wisconsin Oncology Network performed a small Phase II study to assess the use of a modified R-hyperCVAD regimen - one that did not have alternating MA cycles and instead included a maintenance program using rituximab. ${ }^{13}$ This study demonstrated that modified R-hyperCVAD produced overall response rates (ORRs) comparable to the original regimen and had a lower incidence of adverse events despite the study's inclusion of an older patient population. Recently, a retrospective study observing patients with MCL treated at a single center over time concluded that less doseintense therapy (eg, R-CHOP) may yield similar rates of OS as R-hyperCVAD/MA, despite higher rates of initial response to dose-intense therapy. ${ }^{14}$

The role of high-dose chemotherapy followed by autologous hematopoietic stem cell transplantation has also been studied in MCL. Initially, it was reserved for treatment of patients with relapsed disease after response to second-line chemotherapy. However, preliminary evidence failed to show long-term remissions using this strategy. ${ }^{15}$ Not surprisingly, given that prognosis worsens with each failed therapy, the subset of patients who had been through fewer lines of therapy seemed to benefit more than those who had received multiple lines of treatment prior to transplantation. ${ }^{16}$ In line with this decrease in efficacy with subsequent treatments, earlier autologous hematopoietic stem cell transplantations following response to frontline therapy have produced positive results in several prospective studies. ${ }^{17,18}$ Of note, patients whose disease achieves complete or partial remission after induction chemotherapy had better rates of OS and progression-free survival (PFS) after autologous hematopoietic stem cell transplantation. ${ }^{19}$ One recent study implemented an intensified induction chemotherapy regimen followed by autologous hematopoietic stem cell transplantation in newly diagnosed patients with MCL and found a high rate of PFS at 2 years, providing further encouraging evidence for the use of upfront 
transplantations in MCL. ${ }^{20}$ New data on allogeneic and nonmyeloablative stem cell transplantations are adding to the already expanding choices of transplant schemas in MCL; however, their discussion is beyond the scope of this review.

\section{Relapsed or refractory $\mathrm{MCL}$}

Despite favorable responses to chemotherapy in de novo MCL, most patients will experience a recurrence of their disease. Treating patients with relapsed or refractory disease presents a challenge because response rates are lower in this setting than they are in frontline treatments. Treatment options in the second-line setting have historically been limited, with the use of multiagent chemotherapy regimens showing only marginal utility. However, with the advent of newer agents and targeted therapies, the options are expanding. Bortezomib, the first in its class of proteasome inhibitors, was the first agent to be approved by the United States Food and Drug Administration (FDA) for use in relapsed or refractory MCL. ${ }^{21-24}$ Bendamustine, a new agent encompassing properties of both purine analogs and alkylating agents, has been used in this setting as part of combination therapy and has shown promising results. ${ }^{25-28}$ More data are emerging on the use of thalidomide and lenalidomide, two multifunctional immunomodulatory agents, as second-line treatments. ${ }^{29,30}$ Another promising new agent in this setting is temsirolimus, an analog of the immunosuppressant drug sirolimus (first known as rapamycin), which has shown efficacy in treating relapsed or refractory MCL. Table 1 compares the efficacy and general toxicities of the second-line agents currently employed in treating MCL as observed in Phase II trials.

\section{Targeting the PI3K/Akt/mTOR survival pathway}

Rapamycin was isolated from the bacteria Streptomyces hygroscopicus and has been approved by the FDA as an immunosuppressive agent. Since the discovery of rapamycin in the 1970s, the study of its effects on cells has helped scientists explore new intracellular signaling mechanisms, namely those involving the plasma membrane protein phosphatidylinositol 3-kinase (PI3K). The activation of PI3K causes changes within the cell through the phosphorylation of a variety of downstream effector proteins. One of these proteins is Akt, a serine/threonine kinase, which has more than 50 potential downstream targets and is directly activated by PI3K. ${ }^{33}$ One of the most critical proteins affected by Akt is mTOR. Taken together, the PI3K/Akt/mTOR pathway helps control cell metabolism, survival, and proliferation through the activation and inactivation of various proteins, as demonstrated in Figure 1.

Early clinical trials with rapamycin noted the drug's cytostatic properties and its potential as an antitumor agent. ${ }^{34}$ Scientists found that rapamycin exerts its effect on the cell by binding to the $12-\mathrm{kDa}$ immunophilin FK506-binding protein (FKBP12) and that the FKBP12-rapamycin complex inhibits the activity of mTOR..$^{35}$ This prompted an area of research using mTOR inhibitors for anticancer therapy and increased interest in targeting various aspects of the PI3K/ Akt/mTOR pathway.

Current evidence suggests that mTOR exists as a multiprotein complex, with its downstream effects being regulated by its association with various proteins, growth factors, and nutrients. Researchers have isolated two specific complexes,

Table I The comparative efficacy of second-line agents in MCL (data from select Phase II trials)

\begin{tabular}{|c|c|c|c|c|c|}
\hline $\begin{array}{l}\text { Clinical trials in relapsed } \\
\text { or refractory } \mathrm{MCL}\end{array}$ & $\mathbf{N}$ & PR & CR & ORR $^{\mathbf{a}}$ & Significant $\mathbf{A E}^{\mathrm{b}}$ \\
\hline Bortezomib ${ }^{21}$ & $|4|$ & $26 \%(36 / I 4 I)$ & $6 \%(9 / 141)$ & $47 \%$ & Fatigue, thrombocytopenia, neuropathy \\
\hline Bortezomib 22 & 28 & $43 \%(12 / 28)$ & 0 & $46.4 \%$ & \\
\hline Bortezomib 23 & 29 & $21 \%(6 / 29)$ & $21 \%(6 / 29)$ & $41 \%(12 / 29)$ & \\
\hline Bortezomib ${ }^{24}$ & 10 & $40 \%(4 / 10)$ & 0 & $50 \%(5 / 10)$ & \\
\hline Lenalidomide $^{30}$ & 15 & $33 \%(5 / 15)$ & $20 \%(3 / 15)$ & $53 \%(8 / 15)$ & Neutropenia, leucopenia, thrombocytopenia \\
\hline Thalidomide $^{29}$ & 16 & $50 \%(8 / 16)$ & $25 \%(4 / 16)$ & $81 \%(13 / 16)$ & Somnolence, neuropathy, constipation \\
\hline Bendamustine + fludarabine ${ }^{25}$ & 9 & $66 \%(6 / 9)$ & $33 \%(3 / 9)$ & $100 \%(9 / 9)$ & Leukopenia, neutropenic fever, \\
\hline Bendamustine + rituximab ${ }^{26}$ & 16 & $25 \%(4 / 16)$ & $50 \%(8 / 16)$ & $75 \%(12 / 16)$ & thrombocytopenia, anemia \\
\hline $\begin{array}{l}\text { Bendamustine }+ \text { rituximab }+ \\
\text { mitoxantrone }^{28}\end{array}$ & 18 & $44 \%(8 / 18)$ & $33 \%(6 / 18)$ & $78 \%(14 / 18)$ & \\
\hline Temsirolimus $^{31}$ & 27 & $37 \%(10 / 27)$ & $4 \%(1 / 27)$ & $41 \%(11 / 27)$ & Thrombocytopenia, fatigue, \\
\hline Temsirolimus $^{32}$ & 34 & $35 \%(12 / 34)$ & $3 \%(1 / 34)$ & $38 \%(13 / 34)$ & hyperglycemia, dyspnea \\
\hline
\end{tabular}

Notes: a ORR based on PR and CR. ${ }^{\mathrm{b}}$ Generally grade 3 or higher.

Abbreviations: $N$, number of patients with $M C L$ evaluable for efficacy; PR, partial response; CR, complete response; ORR, overall response rate; $A E$, adverse events. 


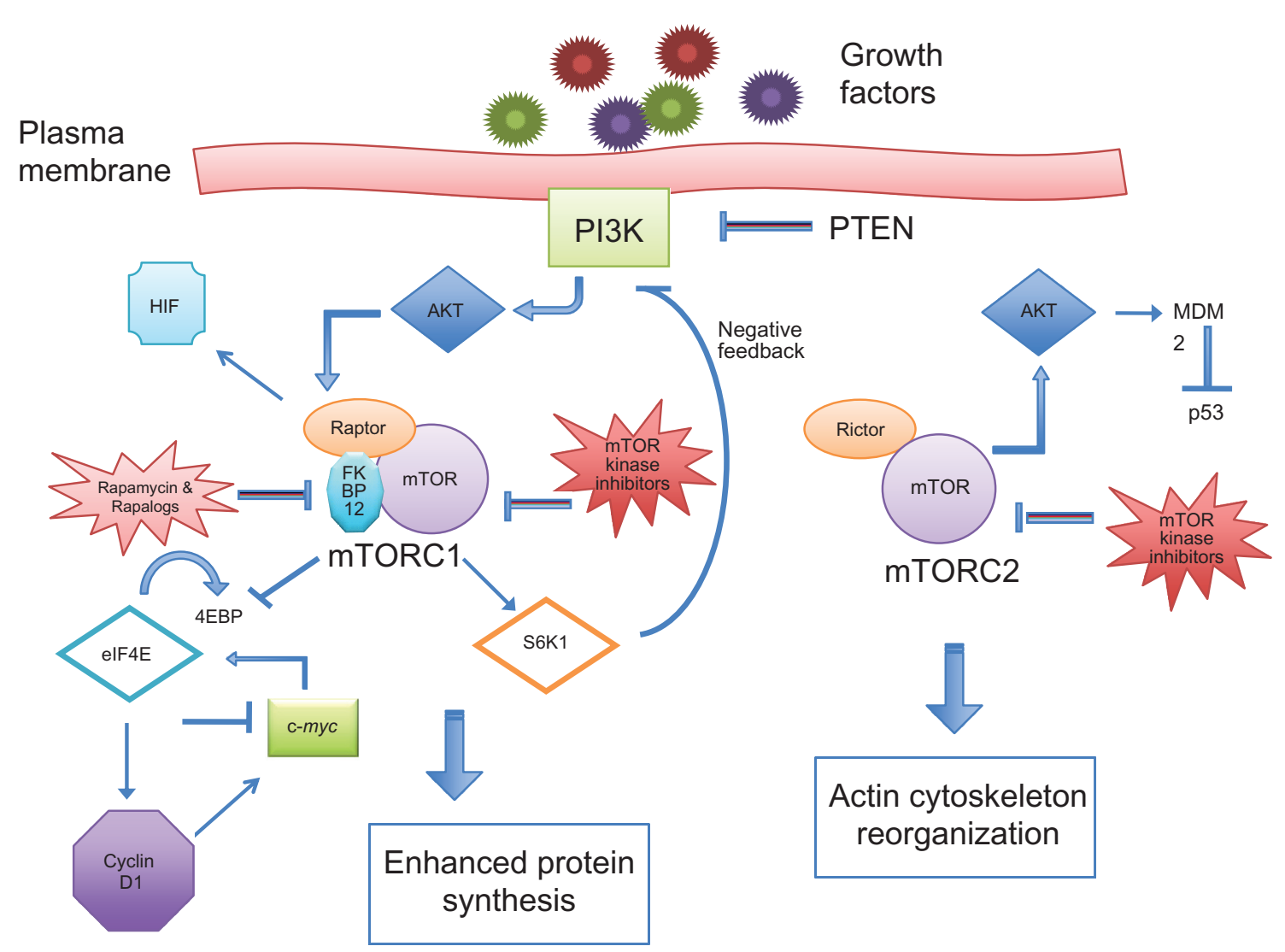

Figure I Intracellular proteins involved in the PI3K/Akt/mTOR pathway.

mTORC1 and mTORC2, each leading a separate branch of the mTOR pathway. The mTORC1 complex includes mTOR and a protein called regulatory-associated protein of mTOR, whereas mTORC2 is made of mTOR and the rapamycininsensitive companion of mTOR protein. ${ }^{36}$ Essentially, activated $\mathrm{mTOR}$ is responsible for integrating cellular signals and triggering downstream targets.

Among other functions, mTORC1 is responsible for the phosphorylation of two substrates - ribosomal p70 S6 kinase (S6K1) and elongation initiation factor 4E (eIF4E)-binding protein 1 (4E-BP1). ${ }^{37}$ Activated S6K1 promotes protein synthesis by allowing translation of ribosomal proteins. The phosphorylation of 4E-BP1 causes disassociation from eIF4E, thus allowing eIF4E to recruit ribosomes to mRNA via association with the mRNA cap. The result of mTORactivated translation of mRNA is the production of proteins that are necessary for G1 cell-cycle progression and initiation of the S phase. ${ }^{38}$ Passage from G1 to S phase is considered the most crucial step in the cell cycle.

Although the exact mechanism by which mTOR regulates cell-cycle progression is not well understood, one proposed mechanism includes the eIF4E-dependent nuclear transport and translation of mRNAs that encode for cell-cycle regulators such as cyclin D1. ${ }^{39,40}$ In general, the cyclins are a group of proteins that guide cell cycling from one phase to another. Four different classes of cyclins exist (A, B, D, and $\mathrm{E}$ ), and each is responsible for regulating different phases of the cell cycle. The role of cyclin D includes promoting the gene transcription involved in the transition from G1 to $S$ phase.

\section{Rationale for targeting mTOR for cancer therapy}

A close study of mTOR's central role in intracellular signaling and advances in research on deregulated signaling pathways in cancer have led scientists to study the effects of aberrant mTOR function. Through years of research, scientists have found correlative data to suggest that mTOR deregulation can lead to tumorigenesis via increased cell growth and survival and via decreased apoptosis.

\section{Deregulation of proteins upstream of mTOR}

In normal cells, mTORC1 activation represses PI3K-Akt signaling upstream via a negative feedback mechanism 
involving S6K1. ${ }^{41}$ In addition, the tumor suppressor gene PTEN can inhibit Akt activation via a reduction in levels of P13K in the cell membrane. ${ }^{42}$ In support of this finding, cells that lack PTEN exhibit a constitutively active form of Akt. ${ }^{43}$ Loss of PTEN from gene alteration or deletion has been found in a variety of human cancers, including lymphoid neoplasms. ${ }^{44,45}$ Other mechanisms of increased PI3K-Akt activity include the amplification of genes that encode for Akt. ${ }^{46}$ An alternate hypothesis suggests that mTORC2 can directly activate Akt, thus circumventing the negative feedback mechanism led by S6K1. ${ }^{47}$

\section{Deregulation of proteins downstream of mTOR}

The overexpression of the downstream protein eIF4E in cancer cells coincides with an increase in tumorigenesis and has become an increasingly vigorous area in oncology research. Scientists have shown that cells exhibiting an overexpression of eIF4E have markedly increased levels of cyclin D1. ${ }^{48}$ One explanation for this finding is an increase in eIF4E-facilitated transport of cyclin D1 mRNA from the cell nucleus to the cytoplasm. ${ }^{49}$ The clinical importance of eIF4E function is supported by evidence in human cancer cells. A strong expression of eIF4E has been observed in some NHL cell lines, particularly in the aggressive and highly aggressive subtypes, suggesting that the level of eIF4E may be predictive of the clinical characteristics and behavior of particular cancers. ${ }^{50}$

Studies on mouse xenografts have demonstrated that in addition to its effects on cell-cycle progression, eIF4E participates in the activation of a pathway that suppresses apoptosis. ${ }^{51}$ In normal cells, mTOR suppresses the DNA repair protein $\mathrm{p} 53$ during periods of cell proliferation. This occurs via the protein MDM2, from the murine double minute $(M D M)$ oncogene, which is a negative regulator of $\mathrm{p} 53$. The normal function of $\mathrm{p} 53$ is to enable DNA repair and trigger apoptosis in cells that cannot be repaired after DNA damage. Elevated levels of eIF4E in oncogenic cells - possibly via the upregulation of mTOR - can lead to an upsurge in transport of MDM2 mRNA from the nucleus. ${ }^{52}$ The enhanced levels of MDM2 can boost p53 inhibition and upset the balance between cell proliferation and programmed cell death, thus leading to tumor growth. ${ }^{52}$

Another group of proteins downstream of mTOR involved in cell growth and proliferation includes those encoded by the $M y c$ family genes: c-Myc, N-Myc, and L-Myc. Studies on murine models have demonstrated that c-Myc enhances protein synthesis during lymphocyte development and carries the ability to induce apoptosis in quiescent cells. ${ }^{53}$ The overexpression of c-Myc has been implicated in the pathogenesis of B-cell lymphomas, especially in more aggressive subtypes. Research has indicated that lymphomagenesis involving c-Myc may be accelerated by various cell-cycle proteins such as cyclin D1. ${ }^{54}$ In addition, elevated levels of eIF4E may further augment the effects of cyclin D1 on cells with deregulated c-Myc. ${ }^{55}$ Studies show that overexpression of eIF4E inhibits c-Myc-induced apoptosis. ${ }^{51}$ Since c-Myc is also an upstream activator of eIF4E, c- $M y c$ may induce a feedback mechanism for suppression of its own proapoptotic function via increased eIF4E. ${ }^{56}$

Hypoxia-inducible factor (HIF) is a family of transcription factors involved in angiogenesis, glucose metabolism, and apoptosis regulation, which are activated under hypoxic conditions in the cell. ${ }^{57}$ Researchers have reported an increase in HIF $1 \alpha$ in hematologic malignancies despite conditions of normoxia. ${ }^{58}$ This increase may be due to the effects of Aktmediated translation of HIF. ${ }^{59}$ Interestingly, the upregulation of HIF $1 \alpha$ was found to correlate with the presence of aberrant mTORC 1 pathways. ${ }^{60}$ It is possible that tumor cells overexpressing Akt may increase HIF1 $\alpha$ expression via the upregulation of mTOR.

\section{Rationale for using mTOR inhibitors for the treatment of $M C L$}

MCL can be differentiated from other subtypes of NHL by the expression of the translocation $\mathrm{t}(11 ; 14)(\mathrm{q} 13 ; \mathrm{q} 32)$ observed by fluorescence in situ hybridization (FISH) and the subsequent overexpression of the cyclin D1 protein as seen by immunohistochemical (IHC) analysis. The $t(11 ; 14)$ determines the deregulated expression of cyclin D1 in lymphoid cells because of its proximity to B-cell immunoglobulin heavy chain transcription enhancers. ${ }^{38}$ Two other types of cyclin D proteins, D2 and D3, also exist, and each is specific to certain tissues. Cyclins D2 and D3 are used in normal lymphoid cells, but are replaced by overexpressed cyclin D1 in cases of MCL. ${ }^{61}$

Various additional proteins have also been shown to be upregulated in MCL. As mentioned earlier, PI3K/Akt is a major signaling pathway that regulates cell growth and cell death through a variety of molecular pathways, including mTOR activation. Interestingly, the PI3K/Akt pathway has been found to be constitutively active in some cases of MCL. In one study, the upregulation of Akt increased the antagonistic activity of phosphorylated MDM2 against p53, which is analogous to earlier preclinical data. ${ }^{62} \mathrm{~A}$ strong correlation between upregulation of Akt and the presence of activated mTOR was also observed, although mTOR 
activation through Akt-independent pathways could not be ruled out. In further support of these data, another study showed the increased activation of the mTOR pathway in MCL cell lines and found enhanced Akt activation in onethird of MCL tumors. ${ }^{63}$ More recent data have indicated that PTEN might not be lost in MCL cells, but rather may persist in an inactivated form secondary to constitutive phosphorylation. ${ }^{64}$ In contrast to earlier studies that predicted that Akt upregulation would be more common in the rare blastoid variant of MCL, new data suggest that Akt activation might be just as common in normal variants of MCL. ${ }^{64}$

Given these findings, the treatment of MCL cells with rapamycin seemed to be the next logical step for researchers. Reports from earlier data demonstrated the effects of rapamycin in significantly reducing cyclin D1 levels in some in vitro cell cultures, presumably by delaying its accumulation. ${ }^{65}$ One study demonstrated a decrease in cyclin D1 mRNA translation secondary to the loss of eIF4E in rapamycintreated cells, which could explain the decrease in cyclin D1 accumulation. ${ }^{40}$ Interestingly, cyclin D1 levels in MCL cells treated with rapamycin were not significantly altered, suggesting that cyclin D1 might not be the primary target of mTOR inhibition in this disease, but rather a downstream effect of mTOR inhibition. ${ }^{66}$ The direct effect of rapamycin on cyclin D1 warrants further study.

What is known about rapamycin is its ability to bind intracellular FKBP12 and form a complex that inhibits the activity of mTORC1. Thus, mTORC1 is also known as the rapamycin-sensitive complex. Since FKBP12-rapamycin is not known to exhibit similar action on mTORC2, the latter is known as the rapamycin-insensitive complex. Scientific interest in mTORC2 inhibition arises partly from its ability to activate Akt signaling and overcome the negative feedback mechanism that normally takes place secondary to PI3K activation.

\section{Clinical data on the use of temsirolimus in $\mathrm{MCL}$}

The National Cancer Institute (NCI) identified rapamycin as a potential anticancer therapy in 1981 and began studying its effects on a variety of human cancers. ${ }^{67}$ The development of rapamycin as an anticancer therapy, however, was limited by its low oral bioavailability (about 14\%) and low solubility in aqueous solutions. ${ }^{68}$ This led to the development of temsirolimus (rapamycin-42-[2,2-bis-(hydroxymethyl)]propionate), a naturally occurring water-soluble ester analog of rapamycin with improved stability and bioavailability when administered intravenously (IV). ${ }^{69}$ The main in vivo metabolite of temsirolimus is rapamycin (sirolimus), and both molecules exhibit similar potency with regard to mTOR inhibition. Similar to rapamycin, temsirolimus binds with high affinity to FKBP12, decreasing phosphorylation of S6K1 and 4E-BP1, inhibiting mRNA synthesis, and resulting in an approximately $15 \%$ decrease in protein synthesis. ${ }^{69}$ Preclinical studies with temsirolimus demonstrated its ability to delay tumor progression in a variety of human cancer models. ${ }^{70-73}$

A dose-escalation study with temsirolimus was undertaken in patients with advanced cancers on the basis of activity of temsirolimus in animal models. ${ }^{74}$ Temsirolimus was administered once weekly at a starting dose of $7.5 \mathrm{mg} / \mathrm{m}^{2}$, and each cycle consisted of four consecutive weekly doses. Rates of immunosuppression were low throughout the study, even in patients receiving the highest dose of $220 \mathrm{mg} / \mathrm{m}^{2}$. The most frequent adverse effects seen were stomatitis, rash, and hypercholesterolemia. The main dose-limiting toxicity of temsirolimus in this study was reversible thrombocytopenia. To find the most appropriate dose for patients, a simulation test was performed to compare the pharmacokinetics between body surface area-based dosing vs fixed/flat dosing. The study showed no difference in the area under the curve and low interpatient variability between the dosing schemas, suggesting that the use of fixed doses would be appropriate for further analyses. ${ }^{74}$

Of the groups of patients with cancer exposed to temsirolimus in early clinical trials, those with renal cell cancer (RCC) appeared to accrue the largest potential benefit from this therapy. A large Phase III randomized trial demonstrated an increase in OS with temsirolimus over interferon alfa, which had been the standard of care for RCC at the time. ${ }^{75}$ In 2008, temsirolimus was FDA approved for the treatment of patients with advanced RCC with poor prognostic features. The approved dose was based on Phase II trials showing the efficacy and safety of weekly infusion with a flat dose of $25 \mathrm{mg}$ delivered IV. ${ }^{76}$ Since the terminal half-life of rapamycin is about three times that of temsirolimus (54.6 hours vs 17.3 hours), the weekly dosing schedule was thought to provide sustained clinical activity between doses. ${ }^{77}$

On the basis of prior research on rapamycin activity in MCL cells, researchers began to assess the effects of singleagent temsirolimus in patients with relapsed MCL. One Phase II trial of temsirolimus for MCL included patients who had relapsed or were refractory to one or more lines of prior therapy. For inclusion in this study, all patients had to have 
cyclin D1-positive tumors as determined by IHC analysis or $\mathrm{t}(11 ; 14)(\mathrm{q} 13 ; \mathrm{q} 32)$ demonstrated by FISH assay as well as measurable disease (defined as a lymph node or tumor mass $\geq 2 \mathrm{~cm}$ or lymphocytosis with an absolute neutrophil count $\geq 5,000$ ). The median age of this study population was 70 years, with most patients having stage IV disease and most having relapsed after an average of four prior lines of therapy. Patients were treated with a flat dose of $250 \mathrm{mg}$ IV once weekly, with each cycle consisting of four doses. The ORR was $38 \%$, with one patient's disease achieving complete response (CR) and twelve patients' diseases achieving partial response [PR; 13 of 34 patients; $90 \%$ confidence interval (CI): $24 \%-54 \%$ ]. The median time to response was 1 month, with eight responses occurring after just one cycle. The median OS was 12 months (95\% CI: 6.7 months to not yet reached) at study completion. Among responders, the median duration of response was 6.9 months (95\% CI: 5.2-12.4 months). Three patients went off study because of intolerable side effects or a perceived decrease in quality of life while on treatment. The most common adverse effects seen in this study were thrombocytopenia, hyperglycemia, anemia, neutropenia, hypertriglyceridemia, mucositis, fatigue, infection, rash, nausea, weight loss, aspartate aminotransferase elevations, dysgeusia, hypercholesterolemia, and sensory neuropathy. Most patients (88\%) required dose reductions secondary to adverse effects, and the study was amended to incorporate dose reductions down to $50 \mathrm{mg}$ IV weekly.

Given the high rate of myelosuppression in the previous study, another Phase II trial was initiated using the FDAapproved dose of $25 \mathrm{mg}$ IV weekly for RCC. The authors analyzed whether adverse effects could be reduced while maintaining efficacy for MCL. As in the previous study, only patients with relapsed or refractory MCL with cyclin D1 positivity and measurable disease were included in this study. Baseline patient characteristics were also similar to those of the previous study, with a median patient age of 69 , stage IV disease in $86 \%$ of patients, and an average of four prior lines of therapy. The ORR was $41 \%$ (11 of 27 patients; 90\% CI: 22\%-61\%) with $1 \mathrm{CR}$ and 10 PRs. Analogous to the previous study, median time to response was 1 month. The median OS was higher in this study at 14 months $(95 \%$ CI: 10-27 months), and the median duration of response for the 11 responders was 6 months (range, 1-26 months). Overall, $70 \%$ of patients needed dose reductions or treatment delays secondary to adverse effects, which was less than that seen in the previous study. Again, thrombocytopenia was the most common side effect and the most common cause of dose reductions, with $82 \%$ of patients experiencing a decrease in platelet counts at the starting dose. Most cases of thrombocytopenia resolved quickly when treatment was delayed by an average of 1 week. The authors concluded that the $25 \mathrm{mg}$ /week dose of temsirolimus had similar efficacy to and better tolerability than higher doses.

To verify a true dose-response relationship, a multicenter Phase III trial compared two doses of temsirolimus, $25 \mathrm{mg}$ and $75 \mathrm{mg}$ IV weekly, against other single-agent therapies chosen by the investigator. ${ }^{78}$ Those chosen by the investigator included agents that had been studied for relapsed MCL, such as thalidomide, lenalidomide, and fludarabine, among others. Patients treated with temsirolimus at the $75 \mathrm{mg}$ dose had a significantly longer PFS (4.8 vs 1.9 months, $P=0.0009 ; 97.5 \%$ CI: $0.25-0.78)$ and higher ORR $(22 \%$ vs $2 \%, P=0.0019$ ) than patients who received one of the investigator's choice therapies. Patients on the 25-mg dose showed a trend toward increased PFS and ORR, but this was not statistically significant at the time of study completion. Median OS did not differ significantly between groups. The only adverse effect that occurred with a significantly higher incidence in the temsirolimus arms than in the investigator's choice therapy arms was thrombocytopenia. Grade 3 or 4 adverse effects occurred more often with the 75-mg dose than with the $25-\mathrm{mg}$ dose ( $89 \%$ vs $80 \%$ ). The authors concluded that there was a dose-response relationship with temsirolimus in MCL based on the differences seen in PFS and overall responses in this study and recommended that $75 \mathrm{mg}$ should be the accepted dose for further studies in MCL. In 2009, the European Commission approved temsirolimus for the treatment of adult patients with relapsed or refractory MCL, based on this trial.

\section{Pharmacokinetics of temsirolimus}

Both temsirolimus and its primary metabolite rapamycin are metabolized hepatically and excreted in the feces. ${ }^{77}$ When temsirolimus is dosed weekly, its concentration decreases to subnanomolar levels within 3-4 days after administration. However, rapamycin remains at therapeutic levels for an extended period of time and likely exerts the primary antitumor effect of temsirolimus. ${ }^{74}$ An in vitro study of the metabolism of temsirolimus demonstrated that exposure to human liver microsomes resulted in the formation of 15 different metabolites; the major enzyme involved in biotransformation of temsirolimus was found to be CYP3A4. ${ }^{79}$ The clinical significance of this finding is that practitioners must be alert to the potential for drug-drug interactions with 
temsirolimus. A study of the effects of administering a potent CYP3A4 inhibitor (in this case, ketoconazole) on systemic exposure to temsirolimus demonstrated the need for a $50 \%$ reduction in the dose of temsirolimus when given together. ${ }^{80}$ A Phase I trial is currently underway to assess the need for temsirolimus dose adjustments in patients with compromised hepatic function.

The clinical studies that led to the approval of temsirolimus for RCC did not include patients with decreased renal function. Since less than $5 \%$ of the drug is cleared renally, the manufacturer suggests that no dose adjustments need be made for renal impairment. ${ }^{77}$ A small study in patients with RCC compared the pharmacokinetic parameters of temsirolimus and rapamycin in patients with normal kidney function with those receiving hemodialysis and did not find a statistically significant difference. ${ }^{81}$ The authors concluded that temsirolimus clearance was not affected by dialysis given its large molecular weight (1,030 Da) and large volume of distribution.

\section{Select adverse effects of temsirolimus in MCL}

Targeted therapies often exhibit a diverse array of side effects not seen with conventional cytotoxic chemotherapy agents, some of which can be linked to their mechanisms of action. For example, hyperlipidemia is commonly seen in patients treated with temsirolimus, the mechanism behind which may be secondary to the inhibition of Akt in adipose tissue by rapamycin. ${ }^{82}$ In normal adipose cells, Akt function is dependent on insulin stimulation and (once activated) can suppress lipolysis. In rapamycin-treated cells, Akt inhibition can result in increased lipolysis and an accumulation of free fatty acids, resulting in the hepatic generation of triglycerides. ${ }^{83}$ Pulmonary toxicity also has been observed in clinical trials with temsirolimus in patients with MCL. ${ }^{31,32,78}$ Symptoms ranged from increased cough or dyspnea to more severe adverse effects, such as grade 3 pleural effusions and hypoxia. In rare cases, a severe adverse reaction known as interstitial pneumonitis has been seen. The mechanism of this toxicity is not well understood, but may be due to an increase in NF-kappaB activation secondary to mTOR inhibition. NF-kappaB is a ubiquitous and inducible heterodimer that mediates the induction of proinflammatory cytokines and thus may explain the pathogenesis of pulmonary toxicity from temsirolimus. ${ }^{84}$ Dermatologic side effects from temsirolimus were most commonly manifested as a maculopapular rash on the face/neck and an acne-like rash on the face and upper chest. A possible explanation for this toxicity could be that mTOR effects epidermal growth factor receptor (EGFR) in normal skin. ${ }^{72}$

\section{Discussion}

Treatment options for MCL have expanded since its classification as a separate and unique disease within NHL in the early 1990s. While therapeutic strategies for frontline therapy are still evolving, the lack of a reliable standard of care makes treating this disease rather challenging. In general, as the number of treatments to which a patient has been exposed increases, the likelihood of a response to additional agents decreases. ${ }^{78}$ Therefore, it is imperative to find efficacious frontline regimens that decrease a patient's likelihood of relapse.

The application of R-CHOP as a standard-of-care treatment in MCL has been disputed given the failure of early studies to show an increase in OS. It was hoped that more dose-intense strategies, such as R-hyperCVAD/MA, would show a clear advantage over R-CHOP, but newer data question this. Given the conflicting evidence, a standard chemotherapy option for frontline treatment for MCL does not exist yet. To establish a standard of care, further research in this area is needed with prospective multicenter comparative trials. Data on early treatment with autologous hematopoietic stem cell transplantation are promising and seem to suggest an OS advantage for patients who respond well to frontline chemotherapy regimens (such as R-CHOP and R-hyperCVAD/MA).

In view of the conflicting data on frontline strategies, the controversy over which therapeutic strategy is most efficacious for newly diagnosed patients will persist. Clinicians are continuing to search for a better understanding of why some patients respond to a given type of treatment when others do not. The use of MIPI scores to predict outcomes is still in the early stages given its relatively new inception, and its utility may not be validated for some time. In the meantime, the search for effective second-line treatments that can prolong survival is becoming increasingly important. With researchers' growing understanding of the molecular pathways involved in the pathogenesis of MCL, the options for second-line treatment are expanding.

An ideal second-line agent would be able to prolong DFS, lacks a maximum cumulative dosage limit so that patients could continue on treatment as long as they respond, and has manageable toxicities. Thus far, the efficacy of second-line agents seems to be similar whether single agents or combination regimens are used, offering average ORRs of $60 \%-70 \%$. Temsirolimus has become a promising therapeutic option in this setting and seems to offer several advantages. The weekly intravenous doses can be given on an outpatient basis as a short infusion, increasing the ease of administration and 
compliance of patients. It does not require dose adjustments for renal dysfunction, which is advantageous in a largely elderly population who may have decreased renal function secondary to comorbidities or prior treatments. Lastly, its unique side-effect profile does not overlap greatly with those of the prior therapies to which this population is likely to have been exposed.

The use of temsirolimus in the frontline setting also warrants investigation. The combination of chemotherapy and rapamycin showed potent activity against Akt-expressing lymphoma cells in murine models and might help in treating cells otherwise resistant to chemotherapy agents such as cyclophosphamide and doxorubicin. ${ }^{85}$ Thrombocytopenia is one of the dose-limiting toxicities for temsirolimus in the MCL population, which is understandable in a population of patients who may have had multiple lines of prior therapy (around four, as noted previously), prior hematopoietic stem cell transplantation, or bone marrow involvement of their lymphoma. The rates of such toxicity might be less frequent in the frontline setting, where patients have healthier and less-suppressed bone marrow.

In addition to temsirolimus, two other rapamycin analogs are currently in clinical trials: everolimus (RAD001) and deferolimus (AP23573). Everolimus is FDA approved for patients with RCC who have progressive disease following first-line therapy. ${ }^{86}$ Its mechanism of action is parallel to that of temsirolimus, and it is available in an oral formulation. In vitro data demonstrate that everolimus inhibits $\mathrm{mTOR}$ activity in MCL cell lines, causes a dose-dependant decrease in phosphorylated 4E-BP1, and results in the enhancement of G1 cell-cycle arrest. ${ }^{87}$ In addition, everolimus exhibited synergistic activity in vitro when combined with other agents used in MCL such as doxorubicin, vincristine, rituximab, and bortezomib. ${ }^{87}$ Deferolimus is currently undergoing Phase II trials in patients with relapsed or refractory hematologic malignancies. ${ }^{88}$ Currently, the optimal use of temsirolimus in MCL is still being elucidated. Researchers are trying to understand mechanisms of resistance to temsirolimus to overcome them and optimize treatment. One mechanism of resistance may be the unexpected upregulation of $\mathrm{mTORC} 2$ corresponding with mTORC1 inhibition by temsirolimus. However, scientists have hypothesized that prolonged exposure to rapamycin may help inhibit both mTORC2 and mTORC1, which warrants the investigation of alternate dosing strategies for temsirolimus to overcome this mechanism of resistance. ${ }^{47}$ Unlike the binding of rapamycin to a preformed mTORC1, the inhibition of mTORC2 may happen prior to its formation. Since both complexes need free
mTOR to form their respective complexes, the inhibition of free mTOR may be the causal factor in mTORC2 inactivation. ${ }^{83}$ Resistance to temsirolimus may also occur through the upregulation of eIF4E independent of mTOR activation. The increase in eIF4E can restore Akt action on the cell membrane and circumvent the effects of mTOR inhibition by temsirolimus. ${ }^{85}$ Therefore, agents directly targeting upstream factors such as Akt deserve investigation and might help overcome this resistance pathway when used in combination with temsirolimus.

A new generation of drugs known as small-molecule mTOR kinase inhibitors is currently in Phase I clinical trials. ${ }^{89}$ Unlike rapamycin analogs that require binding to FKBP12, these agents exert their effects by directly inhibiting mTOR kinase and blocking the formation of both mTORC1 and mTORC2. Among this class of agents are the dual inhibitors of mTOR kinase and PI3K. By targeting various components of the PI3K/Akt/mTOR pathway, these agents may be able to overcome resistance to rapamycin and increase cytotoxicity.

\section{Conclusion}

The treatment of MCL has evolved greatly in the last decade. The application of targeted therapies in the second-line setting has expanded treatment options for patients with relapsed or refractory disease. The use of mTOR inhibitors is especially promising in view of the data on deregulated $\mathrm{PI} 3 \mathrm{~K} / \mathrm{Akt} / \mathrm{mTOR}$ pathways in MCL. Several new agents in this class are in the pipeline, and new dosing strategies for existing agents are still being explored. Thus far, temsirolimus has shown clinical efficacy in this patient population as a single-agent treatment with a favorable side-effect profile. Further research is needed to study this drug in combination with other targeted agents and chemotherapy. Finding efficacious treatments for relapsed or refractory disease is fundamental to increasing survival rates and durations for patients with this rare and aggressive disease.

\section{Disclosure}

The authors report no conflicts of interest in this work.

\section{References}

1. A clinical evaluation of the International Lymphoma Study Group classification of non-Hodgkin's lymphoma. The Non-Hodgkin's Lymphoma Classification Project. Blood. 1997;89:3909-3918.

2. Velders GA, Kluin-Nelemans JC, De Boer CJ, et al. Mantle-cell lymphoma: a population-based clinical study. J Clin Oncol. 1996;14: $1269-1274$.

3. Argatoff LH, Connors JM, Klasa RJ, Horsman DE, Gascoyne RD Mantle cell lymphoma: a clinicopathologic study of 80 cases. Blood. 1997;89:2067-2078. 
4. Samaha H, Dumontet C, Ketterer N, et al. Mantle cell lymphoma: a retrospective study of 121 cases. Leukemia. 1998;12:1281-1287.

5. Bosch F, Lopez-Guillermo A, Campo E, et al. Mantle cell lymphoma: presenting features, response to therapy, and prognostic factors. Cancer. 1998; $82: 567-575$

6. Herrmann A, Hoster E, Zwingers T, et al. Improvement of overall survival in advanced stage mantle cell lymphoma. J Clin Oncol. 2009; 27:511-518

7. Hoster E, Dreyling M, Klapper W, et al. A new prognostic index (MIPI) for patients with advanced-stage mantle cell lymphoma. Blood. 2008; 111:558-565.

8. Martin P, Chadburn A, Christos P, et al. Outcome of deferred initial therapy in mantle-cell lymphoma. J Clin Oncol. 2009;27:1209-1213.

9. Lenz G, Dreyling M, Hoster E, et al. Immunochemotherapy with rituximab and cyclophosphamide, doxorubicin, vincristine, and prednisone significantly improves response and time to treatment failure, but not long-term outcome in patients with previously untreated mantle cell lymphoma: results of a prospective randomized trial of the German Low Grade Lymphoma Study Group (GLSG). J Clin Oncol. 2005;23:1984-1992.

10. Romaguera JE, Fayad L, Rodriguez MA, et al. High rate of durable remissions after treatment of newly diagnosed aggressive mantlecell lymphoma with rituximab plus hyper-CVAD alternating with rituximab plus high-dose methotrexate and cytarabine. J Clin Oncol. 2005;23:7013-7023.

11. Fayad L, Thomas D, Romaguera J. Update of the M. D. Anderson Cancer Center experience with hyper-CVAD and rituximab for the treatment of mantle cell and Burkitt-type lymphomas. Clin Lymphoma Myeloma. 2007;8 Supp1 2:S57-S62.

12. Epner EM, Unger J, Miller T, et al. A multi-center trial of hyperCVAD + rituxan in patients with newly diagnosed aggressive mantle cell lymphoma. ASH Annual Meeting Abstracts. Blood. 2007; 110:A387.

13. Kahl BS, Longo WL, Eickhoff JC, et al. Maintenance rituximab following induction chemoimmunotherapy may prolong progression-free survival in mantle cell lymphoma: a pilot study from the Wisconsin Oncology Network. Ann Oncol. 2006;17:1418-1423.

14. Martin P, Chadburn A, Christos P, et al. Intensive treatment strategies may not provide superior outcomes in mantle cell lymphoma: overall survival exceeding 7 years with standard therapies. Ann Oncol. 2008;19:1327-1330.

15. Freedman AS, Neuberg D, Gribben JG, et al. High-dose chemoradiotherapy and anti-B-cell monoclonal antibody-purged autologous bone marrow transplantation in mantle-cell lymphoma: no evidence for long-term remission. J Clin Oncol. 1998;16:13-18.

16. Vose JM, Bierman PJ, Weisenburger DD, et al. Autologous hematopoietic stem cell transplantation for mantle cell lymphoma. Biol Blood Marrow Transplant. 2000;6:640-645.

17. Geisler CH, Kolstad A, Laurell A, et al. Long-term progression-free survival of mantle cell lymphoma after intensive front-line immunochemotherapy with in vivo-purged stem cell rescue: a nonrandomized phase 2 multicenter study by the Nordic Lymphoma Group. Blood. 2008; 112:2687-2693

18. Tam CS, Bassett R, Ledesma C, et al. Mature results of the M. D. Anderson Cancer Center risk-adapted transplantation strategy in mantle cell lymphoma. Blood. 2009;113:4144-4152.

19. Till BG, Gooley TA, Crawford N, et al. Effect of remission status and induction chemotherapy regimen on outcome of autologous stem cell transplantation for mantle cell lymphoma. Leuk Lymphoma. 2008;49:1062-1073.

20. Damon LE, Johnson JL, Niedzwiecki D, et al. Immunochemotherapy and autologous stem-cell transplantation for untreated patients with mantle-cell lymphoma: CALGB 59909. J Clin Oncol. 2009;27: 6101-6108.

21. Fisher RI, Bernstein SH, Kahl BS, et al. Multicenter phase II study of bortezomib in patients with relapsed or refractory mantle cell lymphoma. J Clin Oncol. 2006;24:4867-4874.
22. Belch A, Kouroukis CT, Crump M, et al. A phase II study of bortezomib in mantle cell lymphoma: the National Cancer Institute of Canada Clinical Trials Group trial IND.150. Ann Oncol. 2007;18: $116-121$.

23. Goy A, Younes A, McLaughlin P, et al. Phase II study of proteasome inhibitor bortezomib in relapsed or refractory B-cell non-Hodgkin's lymphoma. J Clin Oncol. 2005;23:667-675.

24. O'Connor OA, Wright J, Moskowitz C, et al. Phase II clinical experience with the novel proteasome inhibitor bortezomib in patients with indolent non-Hodgkin's lymphoma and mantle cell lymphoma. J Clin Oncol. 2005;23:676-684.

25. Koenigsmann M, Knauf W, Herold M, et al. Fludarabine and bendamustine in refractory and relapsed indolent lymphoma - a multicenter phase I/II trial of the East German Society of Hematology And Oncology (OSHO). Leuk Lymphoma. 2004;45:1821-1827.

26. Rummel MJ,Al-Batran SE, Kim SZ, et al. Bendamustine plus rituximab is effective and has a favorable toxicity profile in the treatment of mantle cell and low-grade non-Hodgkin's lymphoma. J Clin Oncol. 2005;23: 3383-3389.

27. Herold M, Schulze A, Niederwieser D, et al. Bendamustine, vincristine and prednisone (BOP) versus cyclophosphamide, vincristine and prednisone (COP) in advanced indolent non-Hodgkin's lymphoma and mantle cell lymphoma: results of a randomised phase III trial (OSHO\# 19). J Cancer Res Clin Oncol. 2006;132:105-112.

28. Weide R, Hess G, Koppler H, et al. High anti-lymphoma activity of bendamustine/mitoxantrone/rituximab in rituximab pretreated relapsed or refractory indolent lymphomas and mantle cell lymphomas. A multicenter phase II study of the German Low Grade Lymphoma Study Group (GLSG). Leuk Lymphoma. 2007;48:1299-1306.

29. Kaufmann H, Raderer M, Wohrer S, et al. Antitumor activity of rituximab plus thalidomide in patients with relapsed/refractory mantle cell lymphoma. Blood. 2004;104:2269-2271.

30. Habermann TM, Lossos IS, Justice G, et al. Lenalidomide oral monotherapy produces a high response rate in patients with relapsed or refractory mantle cell lymphoma. Br J Haematol. 2009;145:344-349.

31. Ansell SM, Inwards DJ, Rowland KM Jr, et al. Low-dose, single-agent temsirolimus for relapsed mantle cell lymphoma: a phase 2 trial in the North Central Cancer Treatment Group. Cancer. 2008;113:508-514.

32. Witzig TE, Geyer SM, Ghobrial I, et al. Phase II trial of single-agent temsirolimus (CCI-779) for relapsed mantle cell lymphoma. J Clin Oncol. 2005;23:5347-5356.

33. Georgakis GV, Younes A. From Rapa Nui to rapamycin: targeting PI3K/Akt/mTOR for cancer therapy. Expert Rev Anticancer Ther. 2006;6:131-140.

34. Eng CP, Sehgal SN, Vezina C. Activity of rapamycin (AY-22,989) against transplanted tumors. J Antibiot (Tokyo). 1984;37:1231-1237.

35. Bjornsti MA, Houghton PJ. The TOR pathway: a target for cancer therapy. Nat Rev Cancer. 2004;4:335-348.

36. Guertin DA, Sabatini DM. Defining the role of mTOR in cancer. Cancer Cell. 2007;12:9-22.

37. Robert F, Pelletier J. Translation initiation: a critical signalling node in cancer. Expert Opin Ther Targets. 2009;13:1279-1293.

38. Bertoni F, Zucca E, Cotter FE. Molecular basis of mantle cell lymphoma. Br J Haematol. 2004;124:130-140.

39. Rousseau D, Kaspar R, Rosenwald I, Gehrke L, Sonenberg N. Translation initiation of ornithine decarboxylase and nucleocytoplasmic transport of cyclin D1 mRNA are increased in cells overexpressing eukaryotic initiation factor 4E. Proc Natl Acad Sci U S A. 1996;93: 1065-1070.

40. Averous J, Fonseca BD, Proud CG. Regulation of cyclin D1 expression by mTORC1 signaling requires eukaryotic initiation factor 4E-binding protein 1. Oncogene. 2008;27:1106-1113.

41. Manning BD. Balancing Akt with S6K: implications for both metabolic diseases and tumorigenesis. J Cell Biol. 2004;167:399-403.

42. Manning BD, Logsdon MN, Lipovsky AI, Abbott D, Kwiatkowski DJ, Cantley LC. Feedback inhibition of Akt signaling limits the growth of tumors lacking Tsc2. Genes Dev. 2005;19:1773-1778. 
43. Stambolic V, Suzuki A, de la Pompa JL, et al. Negative regulation of $\mathrm{PKB} /$ Akt-dependent cell survival by the tumor suppressor PTEN. Cell. 1998;95:29-39.

44. Sansal I, Sellers WR. The biology and clinical relevance of the PTEN tumor suppressor pathway. J Clin Oncol. 2004;22:2954-2963.

45. Sakai A, Thieblemont C, Wellmann A, Jaffe ES, Raffeld M. PTEN gene alterations in lymphoid neoplasms. Blood. 1998;92:3410-3415.

46. Thompson JE, Thompson CB. Putting the rap on Akt. J Clin Oncol. 2004;22:4217-4226.

47. Guertin DA, Sabatini DM. An expanding role for mTOR in cancer. Trends Mol Med. 2005;11:353-361.

48. Rosenwald IB, Lazaris-Karatzas A, Sonenberg N, Schmidt EV. Elevated levels of cyclin D1 protein in response to increased expression of eukaryotic initiation factor 4E. Mol Cell Biol. 1993;13:7358-7363.

49. Rosenwald IB, Kaspar R, Rousseau D, et al. Eukaryotic translation initiation factor $4 \mathrm{E}$ regulates expression of cyclin D1 at transcriptional and post-transcriptional levels. J Biol Chem . 1995;270:21176-21180.

50. Wang S, Rosenwald IB, Hutzler MJ, et al. Expression of the eukaryotic translation initiation factors $4 \mathrm{E}$ and 2alpha in non-Hodgkin's lymphomas. Am J Pathol. 1999;155:247-255.

51. Polunovsky VA, Rosenwald IB, Tan AT, et al. Translational control of programmed cell death: eukaryotic translation initiation factor 4E blocks apoptosis in growth-factor-restricted fibroblasts with physiologically expressed or deregulated Myc. Mol Cell Biol. 1996;16: 6573-6581.

52. Moller MB, Nielsen O, Pedersen NT. Oncoprotein MDM2 overexpression is associated with poor prognosis in distinct non-Hodgkin's lymphoma entities. Mod Pathol. 1999;12:1010-1016.

53. Iritani BM, Eisenman RN. c-Myc enhances protein synthesis and cell size during B lymphocyte development. Proc Natl Acad Sci U S A. 1999;96:13180-13185.

54. Haupt Y, Harris AW, Adams JM. Retroviral infection accelerates T lymphomagenesis in $\mathrm{E}$ mu-N-ras transgenic mice by activating c-myc or N-myc. Oncogene. 1992;7:981-986.

55. Tan A, Bitterman P, Sonenberg N, Peterson M, Polunovsky V. Inhibition of Myc-dependent apoptosis by eukaryotic translation initiation factor 4E requires cyclin D1. Oncogene. 2000;19:1437-1447.

56. Ruggero D, Montanaro L, Ma L, et al. The translation factor eIF-4E promotes tumor formation and cooperates with c-Myc in lymphomagenesis. Nat Med. 2004;10:484-486.

57. Bacon AL, Harris AL. Hypoxia-inducible factors and hypoxic cell death in tumour physiology. Ann Med. 2004;36:530-539.

58. Zhong H, Mabjeesh N, Willard M, Simons J. Nuclear expression of hypoxia-inducible factor 1alpha protein is heterogeneous in human malignant cells under normoxic conditions. Cancer Lett. 2002;181: 233-238

59. Mazure NM, Chen EY, Laderoute KR, Giaccia AJ. Induction of vascular endothelial growth factor by hypoxia is modulated by a phosphatidylinositol 3-kinase/Akt signaling pathway in Ha-ras-transformed cells through a hypoxia inducible factor-1 transcriptional element. Blood. 1997;90:3322-3331.

60. Dunlop EA, Tee AR. Mammalian target of rapamycin complex 1: signalling inputs, substrates and feedback mechanisms. Cell Signal. 2009;21:827-835.

61. Ott MM, Bartkova J, Bartek J, et al. Cyclin D1 expression in mantle cell lymphoma is accompanied by downregulation of cyclin D3 and is not related to the proliferative activity. Blood. 1997;90:3154-3159.

62. Rudelius M, Pittaluga S, Nishizuka S, et al. Constitutive activation of Akt contributes to the pathogenesis and survival of mantle cell lymphoma. Blood. 2006;108:1668-1676.

63. Peponi E, Drakos E, Reyes G, Leventaki V, Rassidakis GZ, Medeiros LJ. Activation of mammalian target of rapamycin signaling promotes cell cycle progression and protects cells from apoptosis in mantle cell lymphoma. Am J Pathol. 2006;169:2171-2180.

64. Dal Col J, Zancai P, Terrin L, et al. Distinct functional significance of Akt and mTOR constitutive activation in mantle cell lymphoma. Blood. 2008;111:5142-5151.
65. Hashemolhosseini S, Nagamine Y, Morley SJ, Desrivieres S, Mercep L, Ferrari S. Rapamycin inhibition of the G1 to S transition is mediated by effects on cyclin D1 mRNA and protein stability. J Biol Chem. 1998;273:14424-14429.

66. Hipp S, Ringshausen I, Oelsner M, Bogner C, Peschel C, Decker T. Inhibition of the mammalian target of rapamycin and the induction of cell cycle arrest in mantle cell lymphoma cells. Haematologica. 2005; 90:1433-1434.

67. Douros J, Suffness M. New antitumor substances of natural origin. Cancer Treat Rev. 1981;8:63-87.

68. Kahan BD, Camardo JS. Rapamycin: clinical results and future opportunities. Transplantation. 2001;72:1181-1193.

69. Dudkin L, Dilling MB, Cheshire PJ, et al. Biochemical correlates of mTOR inhibition by the rapamycin ester CCI-779 and tumor growth inhibition. Clin Cancer Res. 2001;7:1758-1764.

70. Grunwald V, DeGraffenried L, Russel D, Friedrichs WE, Ray RB, Hidalgo M. Inhibitors of mTOR reverse doxorubicin resistance conferred by PTEN status in prostate cancer cells. Cancer Res. 2002;62: 6141-6145.

71. Geoerger B, Kerr K, Tang CB, et al. Antitumor activity of the rapamycin analog CCI-779 in human primitive neuroectodermal tumor/ medulloblastoma models as single agent and in combination chemotherapy. Cancer Res. 2001;61:1527-1532.

72. Yu K, Toral-Barza L, Discafani C, et al. mTOR, a novel target in breast cancer: the effect of CCI-779, an mTOR inhibitor, in preclinical models of breast cancer. Endocr Relat Cancer. 2001;8:249-258.

73. Shi Y, Gera J, Hu L, et al. Enhanced sensitivity of multiple myeloma cells containing PTEN mutations to CCI-779. Cancer Res. 2002;62: 5027-5034.

74. Raymond E, Alexandre J, Faivre S, et al. Safety and pharmacokinetics of escalated doses of weekly intravenous infusion of CCI-779, a novel mTOR inhibitor, in patients with cancer. J Clin Oncol. 2004;22: 2336-2347.

75. Hudes G, Carducci M, Tomczak P, et al. Temsirolimus, interferon alfa, or both for advanced renal-cell carcinoma. N Engl J Med. 2007;356: 2271-2281.

76. Atkins MB, Hidalgo M, Stadler WM, et al. Randomized phase II study of multiple dose levels of CCI-779, a novel mammalian target of rapamycin kinase inhibitor, in patients with advanced refractory renal cell carcinoma. J Clin Oncol. 2004;22:909-918.

77. Torisel [package insert]. Philadelphia, PA: Wyeth Pharmaceuticals, Inc.; 2008.

78. Hess G, Herbrecht R, Romaguera J, et al. Phase III study to evaluate temsirolimus compared with investigator's choice therapy for the treatment of relapsed or refractory mantle cell lymphoma. J Clin Oncol. 2009;27:3822-3829.

79. Cai P, Tsao R, Ruppen ME. In vitro metabolic study of temsirolimus: preparation, isolation, and identification of the metabolites. Drug Metab Dispos. 2007;35:1554-1563.

80. Boni JP, Leister C, Burns J, Hug B. Differential effects of ketoconazole on exposure to temsirolimus following intravenous infusion of temsirolimus. Br J Cancer. 2008;98:1797-1802.

81. Lunardi G, Armirotti A, Nicodemo M, et al. Comparison of temsirolimus pharmacokinetics in patients with renal cell carcinoma not receiving dialysis and those receiving hemodialysis: a case series. Clin Ther. 2009;31:1812-1819.

82. Morrisett JD, Abdel-Fattah G, Kahan BD. Sirolimus changes lipid concentrations and lipoprotein metabolism in kidney transplant recipients. Transplant Proc. 2003;35:143S-150S

83. Sarbassov DD, Ali SM, Sengupta S, et al. Prolonged rapamycin treatment inhibits mTORC2 assembly and Akt/PKB. Mol Cell. 2006;22:159-168.

84. Aparicio G, Calvo MB, Medina V, et al. Comprehensive lung injury pathology induced by mTOR inhibitors. Clin Transl Oncol. 2009;11:499-510.

85. Wendel HG, De Stanchina E, Fridman JS, et al. Survival signalling by Akt and eIF4E in oncogenesis and cancer therapy. Nature. 2004;428: 332-337. 
86. Motzer RJ, Escudier B, Oudard S, et al. Efficacy of everolimus in advanced renal cell carcinoma: a double-blind, randomised, placebocontrolled phase III trial. Lancet. 2008;372:449-456.

87. Haritunians T, Mori A, O’Kelly J, Luong QT, Giles FJ, Koeffler HP. Antiproliferative activity of RAD001 (everolimus) as a single agent and combined with other agents in mantle cell lymphoma. Leukemia. 2007;21:333-339.
88. Rizzieri DA, Feldman E, Dipersio JF, et al. A phase 2 clinical trial of deforolimus (AP23573, MK-8669), a novel mammalian target of rapamycin inhibitor, in patients with relapsed or refractory hematologic malignancies. Clin Cancer Res. 2008;14:2756-2762.

89. Dancey J. mTOR signaling and drug development in cancer. Nat Rev Clin Oncol. 2010;7:209-219.

\section{Publish your work in this journal}

OncoTargets and Therapy is an international, peer-reviewed, open access journal focusing on the pathological basis of all cancers, potential targets for therapy and treatment protocols employed to improve the management of cancer patients. The journal also focuses on the impact of management programs and new therapeutic agents and protocols on

\section{Dovepress}

patient perspectives such as quality of life, adherence and satisfaction. The manuscript management system is completely online and includes a very quick and fair peer-review system, which is all easy to use. Visit http://www.dovepress.com/testimonials.php to read real quotes from published authors.

\footnotetext{
Submit your manuscript here: http://www.dovepress.com/oncotargets-and-therapy-journal
} 